\title{
Erratum
}

\section{Theory of thermoelectric power of model semimetals and semiconductors}

\author{
K. Durczewski ${ }^{1}$, M. Ausloos ${ }^{2}$ \\ ${ }^{1}$ Polish Academy of Sciences, Institute for Low Temperature and Structure Research, P.O.B. 937, PL-50-950 Wroclaw, Poland \\ 2 SUPRAS, Institute of Physics, B5, Sart-Tilman, University of Liège, B-4000 Liège, Belgium
}

Z. Phys. B85, 59-69 (1991)

In the paper [1], while calculating the contribution of holes to the trial electric and heat currents $J_{i}, U_{i}(i=1$, 2 ), a mistake in sign has been made. In result not only the formula (19) for the currents has to be corrected, but also the illustrative final results for the thermoelectric power (TEP or more precisely the Seebeck coefficient) and the electrical resistivity shown in Figs. 4 and 5 as functions of temperature. We apologize here for the mistake and present below the correction.

The formula (19) in [1] should read

$J_{1}=-J_{0}(3 / 2)\left[F_{1 / 2}(z)+A F_{1 / 2}\left(z^{\prime}\right)\right]$

$J_{2}=-J_{0}\left(k_{B} T\right)\left[\phi(z)-A \phi\left(z^{\prime}\right)\right]$

$U_{1}=U_{0}\left(k_{B} T\right)\left[\phi(z)-A \phi\left(z^{\prime}\right)\right]=-J_{2} / e$

$U_{2}=U_{0}\left(k_{B} T\right)^{2}\left[\psi(z)+A \psi\left(z^{\prime}\right)\right]$

and the corresponding numerical results are shown in the corrected Figs. 4, 5.

The figures confirm the fact that both TEP and the resistivity depend on the values of the electron and hole chemical potentials. For the parameters examined in [1] (the sound velocity $v_{s}=5 \cdot 10^{4} \mathrm{~m} / \mathrm{s}$, the Debye temperature $T_{D}=300 \mathrm{~K}$ and the assumed parameters of the model band structure - see the caption of Fig. 3) both TEP and the resistivity are monotoneous functions of the temperature. No problems with treating compensated materials occur, i.e., ones of the same number of electrons and holes.

The electron-hole asymmetry is confirmed in the final results presented here in the corrected Figs. 4 and 5. We remind that the solid dash and dotted lines represent TEP and the resistivity for given different values of the number of electron and hole bands, their Fermi energies and effective masses. The solid lines correspond to the values which yield the net number of carries $n=n_{e}-n_{h}$ equal to $\pm 1.33 \cdot 10^{20} \mathrm{~cm}^{-3}$, the dash lines to $\pm 2.63 \cdot 10^{20} \mathrm{~cm}^{-3}$ and

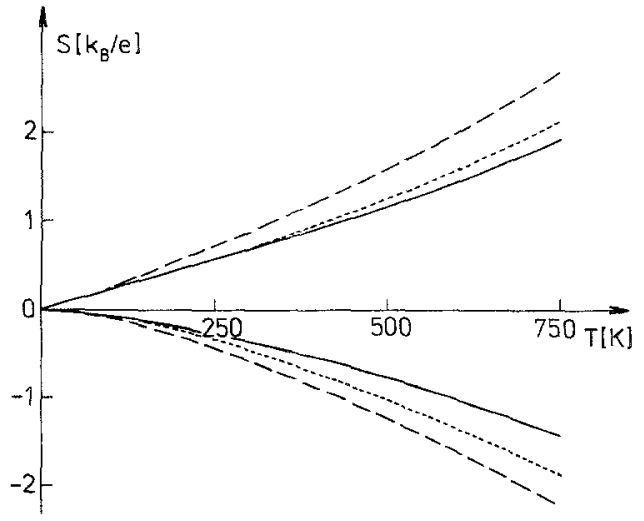

Fig. 4. The diffusion TEP as a function of temperature for model uncompensated semimetal or semiconductor-like materials. It follows from (9) in [1] with the standard acoustic phonon scattering assumed. The parameters of the band structure and the curve marking is the same as in Fig. 3 in [1] (see also present text). For $n_{h}>n_{e}$ they correspond to $S>0$, and for $n_{h}<n_{e}$ to $S<0$. Notice that the electron-hole symmetry of the chemical potential (Fig. 3 in [1]) is broken

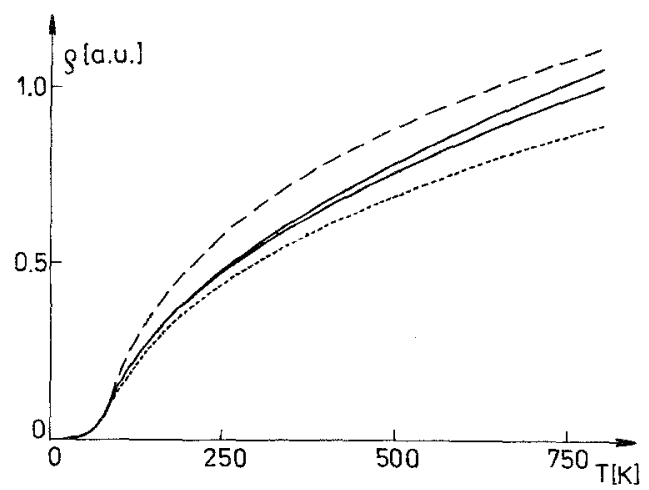

Fig. 5. The dependence of the resistivity (arbitrary units) on the temperature for the same model and material data as in Fig. 4. The value of the resistivity depends on the sign of $n=n_{e}-n_{h}$, what is marked for the solid curves. The upper one corresponds to $n_{e}>n_{h}$. Also for the material constants corresponding to the dotted and dashed curves the greater resistivity is in case $n>0$. The marked dash curve corresponds to $n>0$ and the dotted one to $n<0$ 
the dotted ones to $\pm 6.03 \cdot 10^{20} \mathrm{~cm}^{-3}$. These values follow from (15) and the values given in the caption of Fig. 3 in [1].

The paper by Sondheimer which has been missed in [1] is:

E.H. Sondheimer, Proc. Phys. Soc. (London) 65, 561 (1951).

The paper [20] has been published.
References

1. Durczewski, K., Ausloos, M.: Z. Phys. B85, 59-68 (1991)

20. Paszkowski, S.: Appl. Math. 21, 289 (1991) 